\title{
Intensification of Heat Transfer Processes in the Low Temperature Short Heat Pipes with Laval Nozzle Formed Vapour Channel
}

\author{
Arkady Vladimirovich Seryakov \\ Research Laboratory, LLC Research and Development Company "Rudetransservice”, Veliky Novgorod, Russia \\ Email address: \\ seryakovav@yandex.ru \\ To cite this article: \\ Arkady Vladimirovich Seryakov. Intensification of Heat Transfer Processes in the Low Temperature Short Heat Pipes with Laval Nozzle \\ Formed Vapour Channel. American Journal of Modern Physics. Vol. 7, No. 1, 2018, pp. 48-61. doi: 10.11648/j.ajmp.20180701.16
}

Received: December 6, 2017; Accepted: December 24, 2017; Published: January 11, 2018

\begin{abstract}
The results of flow studies of moist vapour in Laval-liked vapour channels of short linear heat pipes (HPs) are presented. The increase in heat transfer coefficient of short linear HPs, intended for creation the cooling systems of heatstressed designs of spacecraft is carried out by making the HPs vapour channel forms of the Laval-liked nozzle. Comparison of the heat transfer coefficients of short HPs with the standard cylindrical vapour channel and the channel, made in the Laval nozzle form with the equality of all dimensions, flat evaporator shows that the HPs with the Laval-liked nozzle vapour channel exceeds the heat transfer characteristics of the standard HPs with a cylindrical vapour channel under high thermal loads. The study of the flow and condensation in such shaped vapour channels of the short HPs at high thermal loads gives an opportunity to analyze in detail the advantages of using such HPs. Capacitive sensors are additionally installed in cooled top covers of the HPs, and electromagnetic pulses with a frequency of $100 \mathrm{kHz}$ were supplied to them from the external generator. At heating the HPs evaporator, starting from a certain thermal power threshold value, electromagnetic pulses became modulated. It is related with the formations of the boiling process in the capillary-porous evaporator and large amount of vapour over it and its discontinuous distribution. An analytical and numerical evaluation are applied to study the duration of the occurring pulsations, and the analytical results are compared with numerical and experimentally obtained values of the pulsations periods.
\end{abstract}

Keywords: Heat Pipes, Laval Nozzle, Pulsation, Heat Transfer Coefficient

\section{Introduction}

The increase in heat transfer coefficient of short linear HPs is a very important task when designing a spacecraft with a rigid regulation of take-off weight. The application of the linear HPs is justified in the case of the structural impossibility of placing a loop (contoured) HPs and to improve long-term reliability of the cooling systems, which are distributed inlet and outlet closed lines of the loop (contour) HPs.

Pulsating (or oscillating) loop HPs - siphons, was invented in 1990 by Akachi [1]. When the temperature difference between evaporator and condenser exceeds a certain threshold, Tong et al [2], R. Naik, et al [3], the vapour bubbles and condensate liquid plugs begin to oscillate spontaneously back and forth. The amplitude of oscillations is quite strong and the liquid plugs penetrate into both condenser and evaporator. The heat is thus transferred not only by the latent heat transfer liquid-vapour- liquid like in other types of heat pipes, but also by conductive heat transfer of the hot oscillating liquid plugs for cold walls in the condensation zone of loop HPs. This phenomenon is the reason of high heat transfer coefficient of loop HPs in comparison with other types of HPs [4-11].

The increase of the heat transfer processes in short linear open HPs with capillary porous insert is achieved using inner vapour channel made as a Laval-liked nozzle. There is an availability to use a jet vapour nozzle, similar to the Laval nozzle, surrounded by a capillary-porous insert along the full length of the HPs, in case if the heat flow is axially directed, which is typical for short HPs. The shape of this vapour Laval-liked channel, is defined by the inner capillary porous insert. The implementation of the nozzle increases flow 
velocity $[12,20]$, pulse rate of two-phase vapour flow and heat-transfer coefficient in the HPs. Pulsation of the flow in short linear HPs with the capillary porous insert represents more complex phenomena, associated with boiling of the working fluid in the capillary porous evaporator.

Heat-transfer coefficient and operational effectiveness of short linear HPs with capillary-porous inserts and Laval-liked formed vapour channel are defined by closed circulation motion of the working fluid, which undergoes liquid-vapour transition and heat absorption in the evaporation region of the capillary porous insert, vapour phase transfer through a convergent-divergent vapour channel, vapour-liquid transition with heat liberation in the condensation region, and liquid return to the evaporation region through capillaryporous inserts of the HP.

Improvement of the heat-transfer processes in HPs is a complex task, concerning the device's detailed mechanical design and gas dynamics. Flowing vapour, with microdrops of condensate, appears as a nonlinear flow, with attendant internal processes, such as interphasic heat and mass exchange, and power dissipation, Gupta et all [13]. Static regain of supersaturated vapour flow in the diffuser condensation region of the HPs with turbulator, causes flow stagnation, strong vorticity production near the condensation surface and reverse vapour flow [14-17]. Thermal loadrelated processes of vorticity formation and density and pressure fluctuation, proceeding in the convergent-divergent vapour channel of HPs, are of the utmost interest. For a field research of these processes, stainless steel HPs have been manufactured with diethyl ether as the working fluid [18-19].

\section{Materials and Methods}

The diethyl ether $\mathrm{C}_{4} \mathrm{H}_{10} \mathrm{O}$ is selected as the main working fluid, which has the boiling temperature under the atmospheric pressure of $\mathrm{T}_{\mathrm{B}}=308.65 \mathrm{~K}\left(35.5^{\circ} \mathrm{C}\right)$, freezing temperature $\mathrm{T}_{\mathrm{F}}=156.95 \mathrm{~K}\left(-116.2^{\circ} \mathrm{C}\right)$ and critical parameters $\mathrm{T}_{\mathrm{C}}=466.55 \mathrm{~K}\left(193.4^{\circ} \mathrm{C}\right), \mathrm{P}_{\mathrm{C}}=3.61 \mathrm{MPa}$. Successful use of diethyl ether as the working fluid for Wilson chambers, on long liquid phase at $413.15 \mathrm{~K}\left(140^{\circ} \mathrm{C}\right)$, shows its heat resistance, and allows its use as the HP working fluid. The volume of the working fluid in the made of a metal mesh capillary-porous insert and evaporator is not less than $18 \mathrm{~cm}^{3}$. The developed sensor enables us to perform measurements of local characteristics of the film flows primarily, the film thickness and temperature, without making any major disturbances in the flow. To measure the fluid condensate film thickness, short HPs were used whose vapour channel was made in the form of a Laval-liked nozzle [20]. The HPs are described in details below.

The capillary-porous evaporator 7 is constructed of 43 circular layers of thin stainless steel net. Each layer is 0.07 $\mathrm{mm}$ thick with the mesh size of $0.04 \mathrm{~mm}$ and the aggregate thickness of $3 \mathrm{~mm}$; all layers are spot-welded to the flat bottom lid 5 .

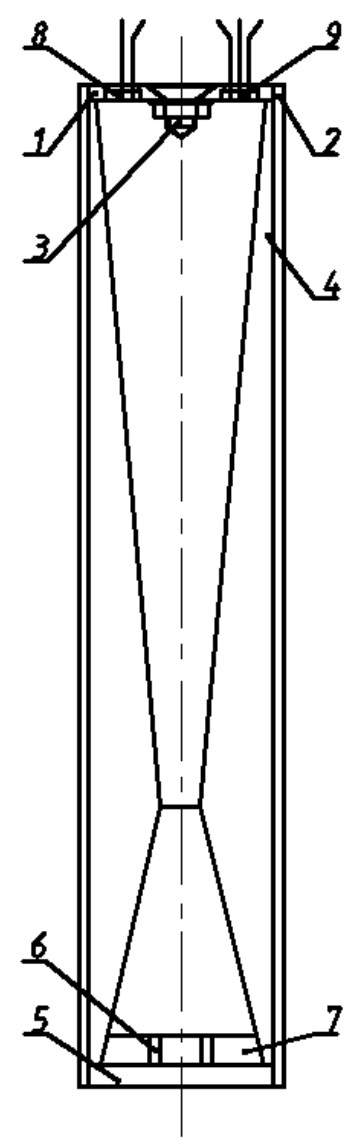

Figure 1. Layout of HP equipped with capacitance sensors: 1 - flat upper lid with a smooth surface; 2 - cylinder body of HP; 3 - cone-shaped turbulence agitator; 4 - capillary-porous insert defining the vapour channel's form; 5 - flat bottom lid; 6 - injector channels; 7 - capillaryporous evaporator; 8, 9-capacitance sensors, one of which is intended for a condensate film thickness measurement, while another one has a sensing element of CT3-19 thermistor mounted on its electrodes to measure the film temperature.

The capillary-porous evaporator 7 is constructed of 43 circular layers of thin stainless steel net. Each layer is 0.07 $\mathrm{mm}$ thick with the mesh size of $0.04 \mathrm{~mm}$ and the aggregate thickness of $3 \mathrm{~mm}$; all layers are spot-welded to the flat bottom lid 5. Outer diameters of the circular layers gradually decrease in such a manner that the lateral side of the assembled evaporator forms a pointed cone with a $45^{\circ}$ total point angle that aligns with the angle of the end part of the capillary-porous insert 4 . The capillary-porous insert 4 is assembled on a dedicated profiled frame made of layers of thin stainless steel net same as the evaporator. Each layer is $0.07 \mathrm{~mm}$ thick with mesh size of $0.04 \mathrm{~mm}$, the total thickness of the insert is $1.5 \mathrm{~mm}$ at the edges and $7.5 \mathrm{~mm}$ at the critical diameter of the inner nozzle. All layers of the insert are radially stitched together along the forming insert with a thin wire $0.05 \mathrm{~mm}$ in diameter and stitch spacing of $5-7 \mathrm{~mm}$. Eight lines of the wire stitches that radially fix the net layers together are tilted by $45^{\circ}$ towards each other and form a rigid structure of the capillary-porous insert with an inner vapour channel made in the form of a nozzle, similar to the Laval nozzle. At the edge of insert 4 that is adjacent to the evaporator 7, an end portion is placed; its total angle is 
somewhat wider than that of the diffuser portion of the vapour channel and equals $45^{\circ}$. The longitudinal length of the end portion is $3 \mathrm{~mm}$ and matches the thickness of the evaporator 7 exactly. The $45^{\circ}$ angle of the end portion of insert 4 is formed by the gradually decreasing net layers' lengths, from the outer layer to the inner one, and matches the angle of the evaporator 7 exactly. The multilayered evaporator with a tapering lateral side is tightly inserted into the rigid capillary-porous insert 4 at the full length of the end portion and is spot-welded to it in 8 spots along the perimeter of the insert. After being cooled down at the boiling temperature of fluid nitrogen $77 \mathrm{~K}\left(-196^{\circ} \mathrm{C}\right)$, the assembled capillary-porous insert, together with the evaporator on the flat bottom lid 5, is tightly inserted in the stainless steel cylindrical shell 2 of the HP.

Porosity of the insert and evaporator is $72 \%$ and together they form one hydraulic system designed to deliver the service fluid to the evaporator when the HP is operating. The HP length is $100 \mathrm{~mm}$, its diameter is $20 \mathrm{~mm}$, the max. diameter of the vapour nozzle in the convergent and divergent regions is $16 \mathrm{~mm}$, the critical nozzle diameter is 4 $\mathrm{mm}$, the length of the nozzle convergent region is $13 \mathrm{~mm}$, the total angle of the convergent region is $41^{\circ}$, the length of the nozzle divergent region is $81 \mathrm{~mm}$, the total angle of the divergent region is $8.5^{\circ}$, and the length of the cylindrical region in the nozzle throat section is $1 \mathrm{~mm}$.

\subsection{Capacitance Sensor}

The compact open capacitance sensor constitutes the following device whose appearance is shown in Figure 2. The sensor's body is made in the form of a flat circular flange 5.5 $\mathrm{mm}$ in diameter and $1 \mathrm{~mm}$ thick, with two cylindrical through bores $1 \mathrm{~mm}$ in diameter each that are arranged symmetrically with respect to the longitudinal axis of the flange, $2 \mathrm{~mm}$ from each other. Each through bore has vacuum-tight glass-to-metal seals $1 \mathrm{~mm}$ in diameter. Two gauging electrodes are tightly fixed in the vacuum-tight glass-to-metal seals, each of them $0.5 \mathrm{~mm}$ in diameter and $10 \mathrm{~mm}$ in length. The space between the gauging electrodes' axes is a=2 $\mathrm{mm}$. A ground electrode $0.5 \mathrm{~mm}$ in diameter and $10 \mathrm{~mm}$ in length is additionally welded to the external surface of the flange.

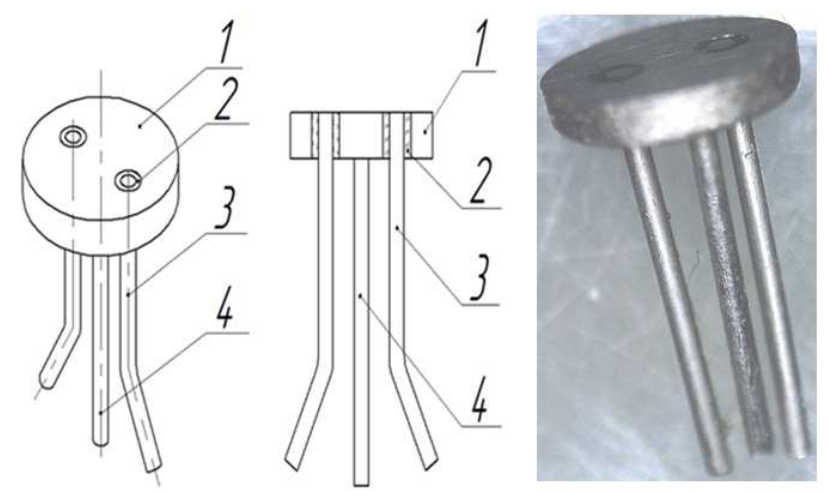

Figure 2. Compact open capacitance sensor layout. 1 - flange with a gauging surface; 2 - glass insulators; 3 - gauging electrodes with gauging end surfaces; 4 - ground electrode. The top surface is polished and called a gauging surface of the sensor.

The flange and gauging and ground electrodes of the open capacitance sensor are made of 29 NK kovar with thermal coefficient of linear expansion of $\sim 5 \cdot 10^{-6} \mathrm{~K}^{-1}$, density of $8350 \mathrm{~kg} / \mathrm{m}^{3}$ and thermal conductivity of $19 \mathrm{~W} / \mathrm{m} \cdot \mathrm{K}$.

All details of the calculations, design, fabrication and calibration of capacitive sensors and a high-frequency capacitive measurement devices are given in [14-19]. The vacuum-tight glass-to-metal seals of capacitance condensation sensors are characterized by high mechanical stability that provides means to sustain the excessive pressure up to 30 bar, and by high air-tightness or low gas permeability (gas leakage) that does not exceed $10^{-9} \mathrm{mbar} \cdot 1 \cdot \mathrm{s}$ ${ }^{1}\left(10^{-5} \mathrm{~Pa} \cdot \mathrm{m}^{3} \cdot \mathrm{s}^{-1}\right)$. The lack of aging materials (plastic masses, adhesives, organic materials, etc.) in the structure of our open capacitance sensors results in the high stability of their features supported by the value permanence of insulation resistance and self-capacitance of the first sensor specimens produced since 1990 .

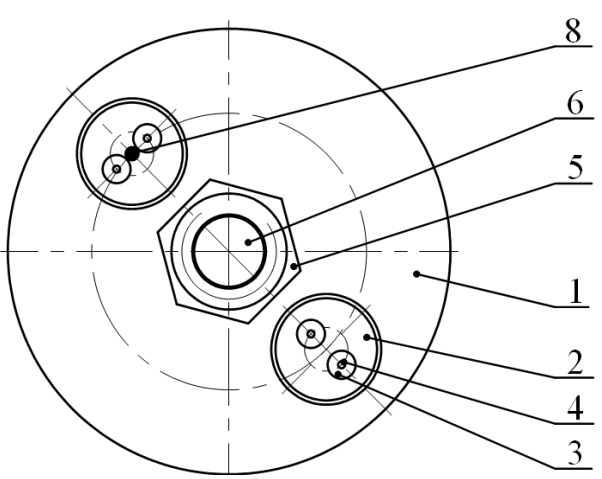

Figure 3. Layout of the installation of two capacitance sensors into the top lid of the HP: 1 - top lid of the HP made of stainless steel 1 X18H9T; 2 - actual capacitance sensors that are laser welded to the lid 1 along their perimeter, herewith the polished gauging surface of sensors is flush-lined with the lid's inner surface; 3 - glass insulators (glass-to-metal seals); 4 -gauging electrodes of both sensors; 5 - fastening nut of the HP filling unit that is welded to the inner surface of the lid 1; 6 - screw plug of the HP filling unit; 7 - capacitance sensor's ground electrode; 8 - micro-thermistor that is a sensing element of thermistor CT3-19 and is laser welded to the tangential oriented end faces of the electrodes of one of the capacitance sensors.

The micro-thermistor is $0.2 \mathrm{~mm}$ in diameter and is electrically insulated from the HP lid. The HP condensing surface of the top lid 1 and radially oriented end faces of the gauging electrodes of the capacitance sensor are also shown here. 


\subsection{Temperature Measurement by Using a Thermistor}

It is well - known used in english-language literature polynomial equation of the third degree by J. S. Steinhart, S. R. Hart [21-22], for a temperature $\mathrm{T}_{\mathrm{SH}}$ calculation by the thermistor and containing linear and cubic in the logarithm of the resistance $\ln _{\mathrm{C}}$ parts:

$$
\frac{1}{\mathrm{~T}_{\mathrm{SH}}}=\mathrm{A}+\mathrm{B} \cdot \ln \mathrm{R}_{\mathrm{C}}+\mathrm{C} \cdot \ln \mathrm{R}_{\mathrm{C}}^{3}
$$

where A, B, C-coefficients of decomposition. The range of application of this equation for temperature measurements with one set of coefficients does not exceed $50 \mathrm{~K}$, and the methodical error of the temperature determination is not less than $5 \cdot 10^{-2} \mathrm{~K}-10^{-1} \mathrm{~K}$ [21-22].

To improve the accuracy and extend the range of temperature measurement with thermistors we developed polynomial equation of the fourth degree [23-26] with a reference temperature to the inflection point of the functional dependence $\ln R_{C}$ from the $\mathrm{T}$ of the thermistors CT3-19. We obtained a quartic polynomial equation [23-26] that connected the temperature $\mathrm{K}$ and the logarithm of the thermistor resistance as follows:

$$
\frac{1}{T}=A_{0}\left(\tau_{d}\right)+A_{1}\left(\tau_{d}\right)\left(\operatorname{lnR}_{C}-7.63_{2}\right)+A_{2}\left(\tau_{d}\right)\left(\operatorname{lnR}_{C}-7.63_{2}\right)^{3}+A_{3}\left(\tau_{d}\right)\left(\operatorname{lnR}_{C}-7.63_{2}\right)^{4}
$$

where $A_{i}\left(\tau_{d}\right)$ is the expansion coefficients with regard to the time drift $\tau_{\mathrm{d}}$, months; the point of minimum of the derivative distribution $\mathrm{d}(1 / \mathrm{T}) / \mathrm{d}\left(\ln _{\mathrm{C}}\right) \cdot 10^{4}, 1 / \mathrm{K}$, as a function of the logarithm of the resistance of the thermistor $\ln R_{\mathrm{C} \text { min }}=7.63_{2}$ \pm 0.01 , which corresponds the inflection point $\mathrm{T}_{\text {inf }}=336.34 \mathrm{~K}$ $\left(63.19^{\circ} \mathrm{C}\right)$, which is calibration invariant of the thermistor CT3-19.
The maximum random error in the measuring of the thermistor resistance is the $5 \cdot 10^{-4} \mathrm{Ohm}$, the error of determination of temperature (difference of temperature) of the thermistor CT3-19 using the equation (2) not more than $(3 \div 5) \cdot 10^{-4} \mathrm{~K}$. The range of temperature measurement with thermistors expanded up to $200 \mathrm{~K}$.

\section{Measurement of the Heat Transfer Coefficient}

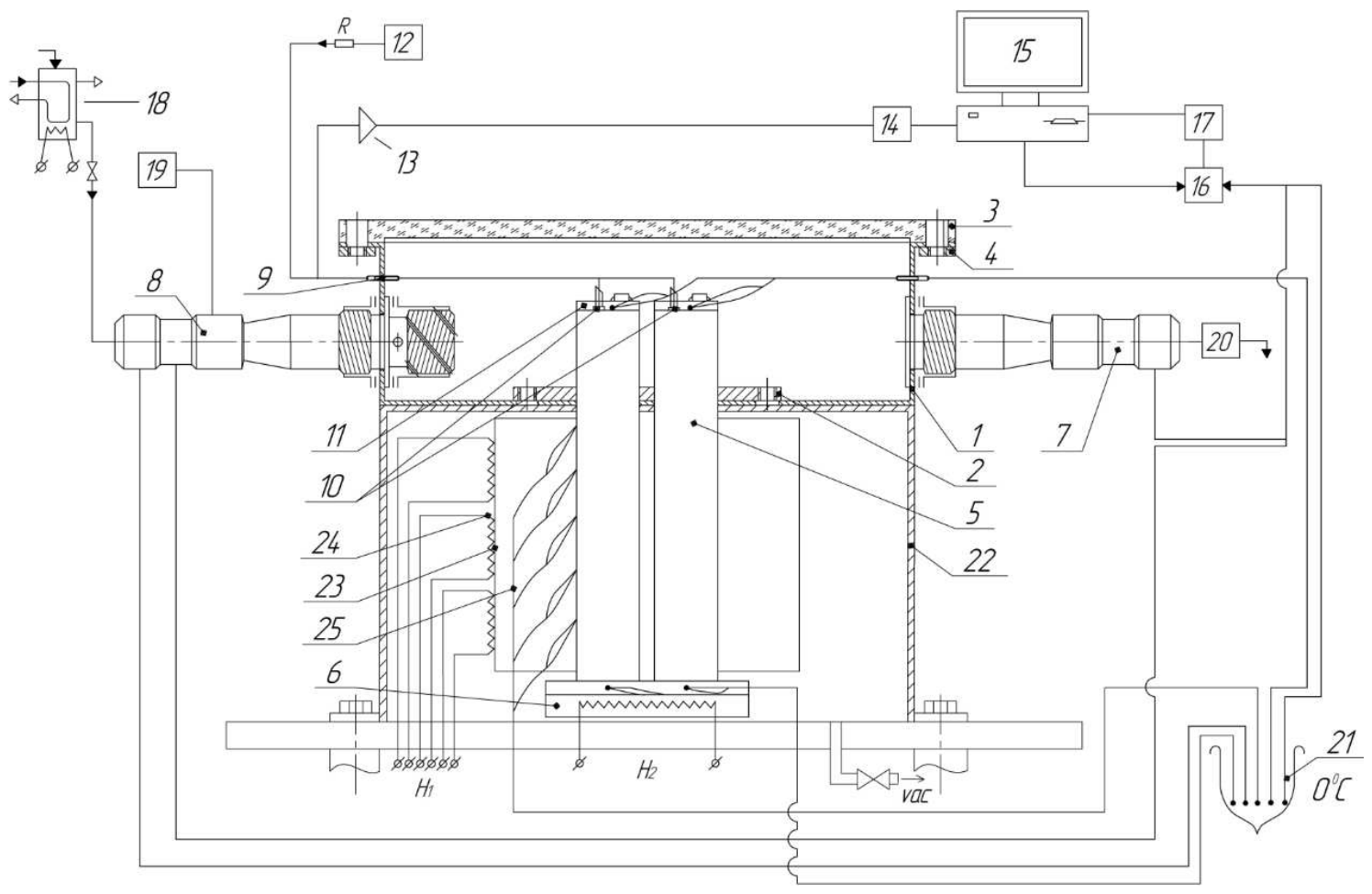

Figure 4. Vortical calorimeter. 1 - vortical continuous-flow calorimeter; 2 - heat pipes bolting flange; 3 - glass cover; 4 - cover fastening; 5 - heat pipes; 6 resistance heater; 7 - outlet stub tube for water flow; 8 - inlet stub tube for water flow; 9 - silicone sealant of the sensing wire; 10 - capacitive sensors for measuring the thickness of the condensed layer of the working fluid; 11- the measuring and reference generators of the capacitive transducer; 12 - external digital generator; 13 - power assist element; 14 - digital oscilloscope; 15 - computer; 16 - commutation switch; 17 - digital voltmeter; 18 - container for constant water head; 19 - source of air bubble; 20 - water flow meter; 21 - vacuum-jacketed zero temperature container; 22 - vacuum chamber; 23 - adiabatic shell; 24 - the three sections of the regulatory heaters; 25-differential thermocouple.

Condensation zones of the HPs are provided with insulated thermocouples and set to a depth of the 1.5 diameter into the vortex continuous-flow calorimeter, shown in Figure 4, with stabilized water flow. To ensure accurate measuring of thermal power and heat removal augmentation in the HPs, jet flow of input water is swirled, values of flow velocity and 
vorticity due to air bubbles are recorded. The HPs evaporators, also equipped with thermocouples, is heated using a resistance heater, and the temperature is maintained at $\delta \mathrm{T}, \mathrm{K}$ higher than the diethyl ether boiling temperature $\mathrm{T}_{\mathrm{B}}$ of $308.65 \mathrm{~K}\left(35.5^{\circ} \mathrm{C}\right)$ under atmospheric pressure. The heater temperature is stabilized and HPs evaporator s overheat value is set in the range of $\delta \mathrm{T}=\mathrm{Tev}-\mathrm{T}_{\mathrm{B}}=0 \div 20 \mathrm{~K}$, herewith thermal power of single HP does not exceed $120 \mathrm{~W}$.

The main HP, called measuring, is filled with diethyl ether and the reference one, which is completely identical to the main HP, is filled with dehumidified air with dew point temperature lower than $233.15 \mathrm{~K}\left(-40^{\circ} \mathrm{C}\right)$. The heat transfer coefficient of the second HP does not exceed $0.15 \%$ from the first one (measuring one) and is not taken into account. The second HP, completely identical to the first one, and performing the function of the reference one is designed for measuring the condensate film thickness in the first, measuring one, filled by diethyl ether [14-17].

To reduce heat loss when working with HPs, they are placed in a stainless steel vacuum chamber 22 , where they are further surrounded by thin-walled copper adiabatic screen 23 , the inner surface of which is covered with a layer of nickel, and on the outer surface placed 3 section guard heaters 24 . The value of nonadiabaticity near the middle of the HP does not exceed $\sim 2 \cdot 10^{-2} \mathrm{~K}$, in the field of the resistive heater $\mathrm{H}_{2}$ and HPs evaporators nonadiabaticity is about $\sim 10^{-1}$ $\mathrm{K}$.

Measurement were performed to disclose variability of the heat transfer coefficients in Laval-liked HPs and with standard cylindrical vapor channel. The heat transfer coefficients in HPs, Faghri [27] is defined by the formula:

$$
\mathrm{K}_{\mathrm{HP}}=\frac{\mathrm{E}}{\mathrm{F}\left(\mathrm{T}_{\mathrm{ev}}-\mathrm{T}_{\text {cond }}\right)}
$$

where $K_{H P}$ - heat transfer coefficient of the HPs, W/K; E thermal power, supplied in the evaporator of the HPs, W; F (z) - surface area of the evaporator inside the vapour channel of the HPs, $\mathrm{m}^{2} ; \mathrm{T}_{\mathrm{ev}}$ - temperature of the evaporator, $\mathrm{K} ; \mathrm{T}_{\text {cond }}$ temperature of the condensation surface, $\mathrm{K}$.

Temperature of evaporator surface and condensation surface of HPs were measured using differential thermocouples copper-constantan, heat power E, W; transferred by condensations zones of HPs to the calorimeter, was defined by the formula:

$$
\mathrm{E}=\mathrm{G} \cdot\left(\mathrm{C}_{\mathrm{H} 2 \mathrm{O}}+\frac{\mathrm{C}_{\mathrm{K}}}{\rho_{\mathrm{H} 2 \mathrm{O}} \mathrm{V}_{0}}\right) \cdot\left[\mathrm{T}_{\mathrm{K}}(\tau)-\mathrm{T}_{\mathrm{K} 0}(\tau)\right]
$$

where $\mathrm{G}$ - water flow in a vortex flow calorimeter, $\mathrm{kg} / \mathrm{s} ; \mathrm{C}_{\mathrm{H} 2 \mathrm{O}}$ - the specific heat of water, $\mathrm{J} / \mathrm{kg} \cdot \mathrm{K}$;

$\mathrm{C}_{\mathrm{K}}$ - the heat capacity of the calorimeter, $\mathrm{J} / \mathrm{K} ; \rho_{\mathrm{H} 2 \mathrm{O}}-$ the density of water in the calorimeter, $\mathrm{kg} / \mathrm{m}^{3}$;

$\mathrm{V}_{0}$ - the internal volume of the calorimeter, $\mathrm{m}^{3} ; \mathrm{T}_{\mathrm{K} 0}(\tau), \mathrm{T}_{\mathrm{K}}$ $(\tau)$ - the water temperature at the inlet and outlet of the calorimeter, K. Input temperature of water at the calorimeter, temperature differences (heat value) of the flowing water at entrance and exit of the calorimeter were also measured using differential thermocouples copper-constantan, comparator P3003 and digital voltmeter V7-34A. Water flow rate was measured using ultrasonic flowmeter. Total measuring inaccuracy of the thermal power E of the HPs does not exceed $1.7 \%$.

Comparison of heat transfer coefficients in Laval-liked HPs and HPs with standard cylindrical vapour channel, with the equality of all dimensions showed following results, presented in Figure 5.

The increase of the heat transfer coefficient in the HP with a Laval - liked vapour channel appears to be related to the emergence of a vortex ring near the condensation surface of the HP.

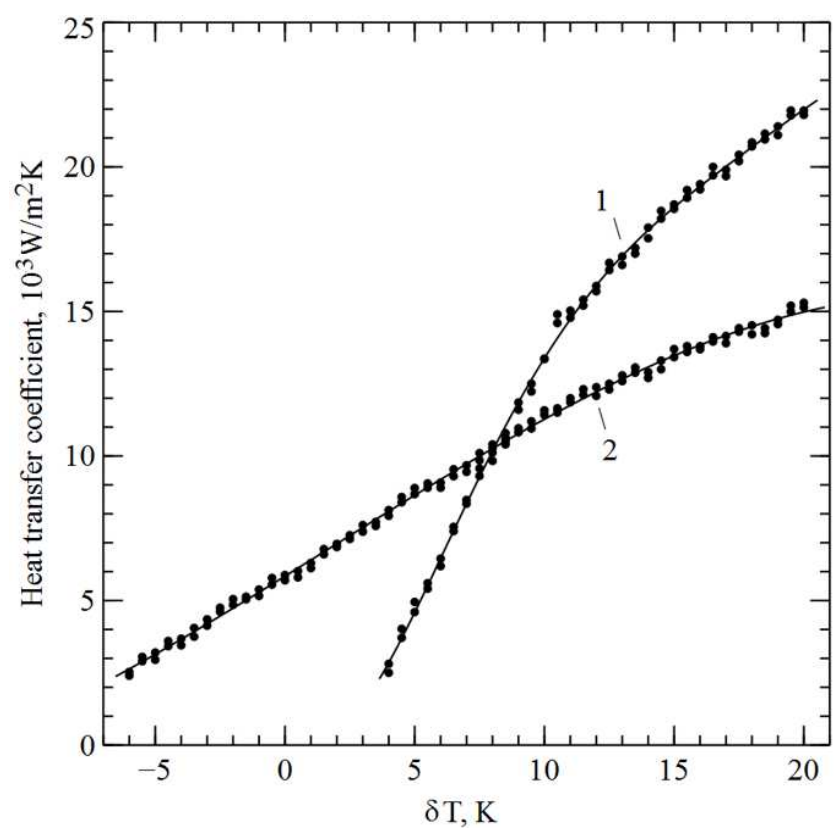

Figure 5. Comparison of heat transfer coefficients in Laval-liked HP and $H P$ with standard cylindrical vapour channel. 1-Laval-liked HPs; 2- HPS with standard cylindrical vapour channel.

\subsection{Numerical Model Details. The Vortex Ring Formation}

Numerical simulations of the vortex flows inside a vapour channel of the Laval-liked HPs have been performed in finite element modeling in CFD 10.0 code Fluent 6.3.26 under 2D, double precision axis-symmetric conditions. Navier-Stokes equations with measured boundary conditions were solved, i.e. using fixed temperature values of heat source and heat outlet. In the construction of the design model about 457233 finite elements were used, with increased meshing at injection capillary channels sections, nozzle throat section and turbulence element. In Figure 6 and Figure 7 shows a diagrams of the vortex obtained by numerical simulation of CFD 10.0 code. 
Velocity Magnitude $-\mathrm{m} / \mathrm{s}$

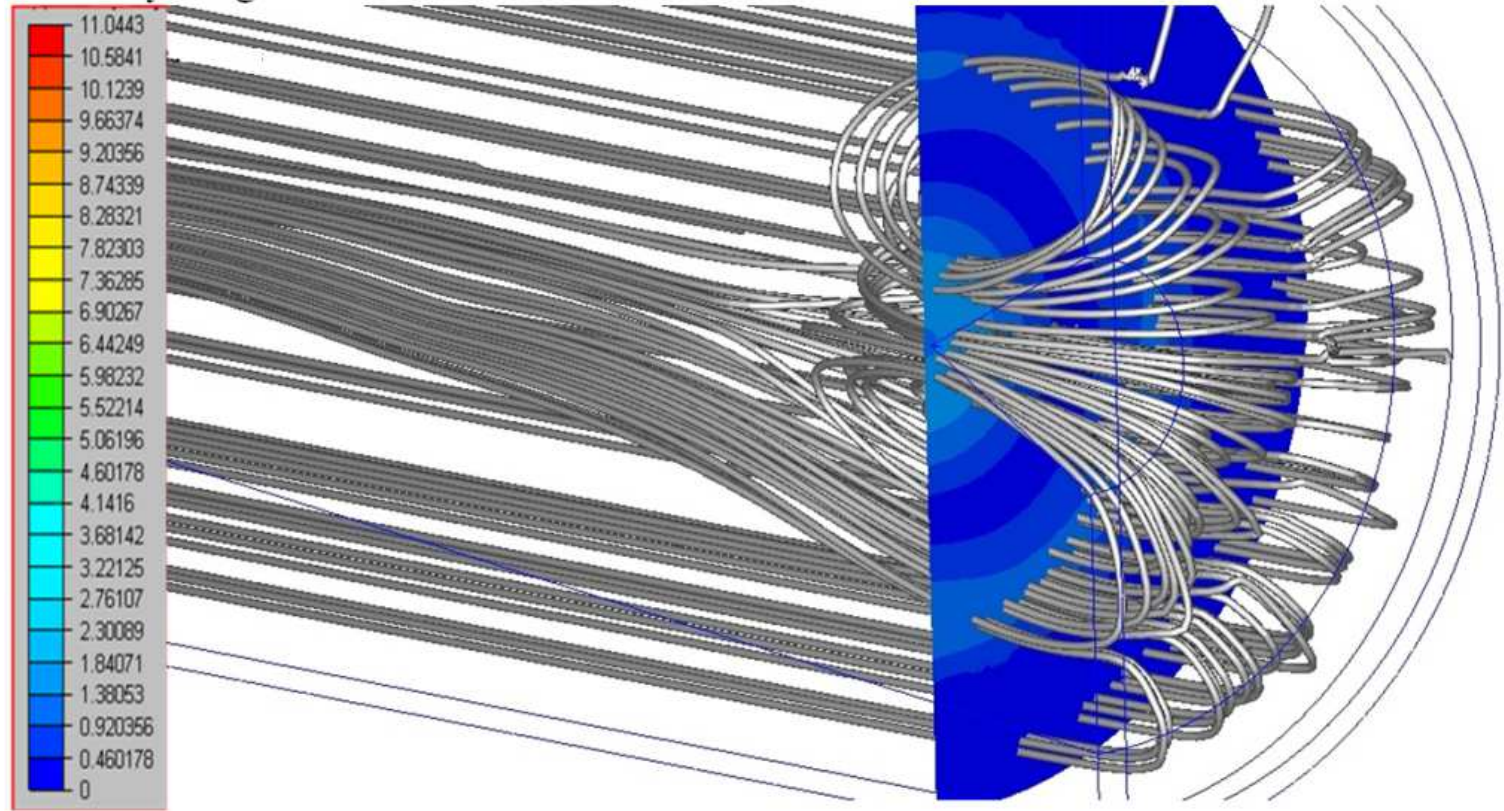

Figure 6. The occurrence of a vortex ring near the condensation surface inside of Laval-liked HPs at low heat loads. Moving vapour jets due to the impact of the Coanda effect sticks to the HPs walls and vortex ring formed from the periphery to the center of the vapour channel.

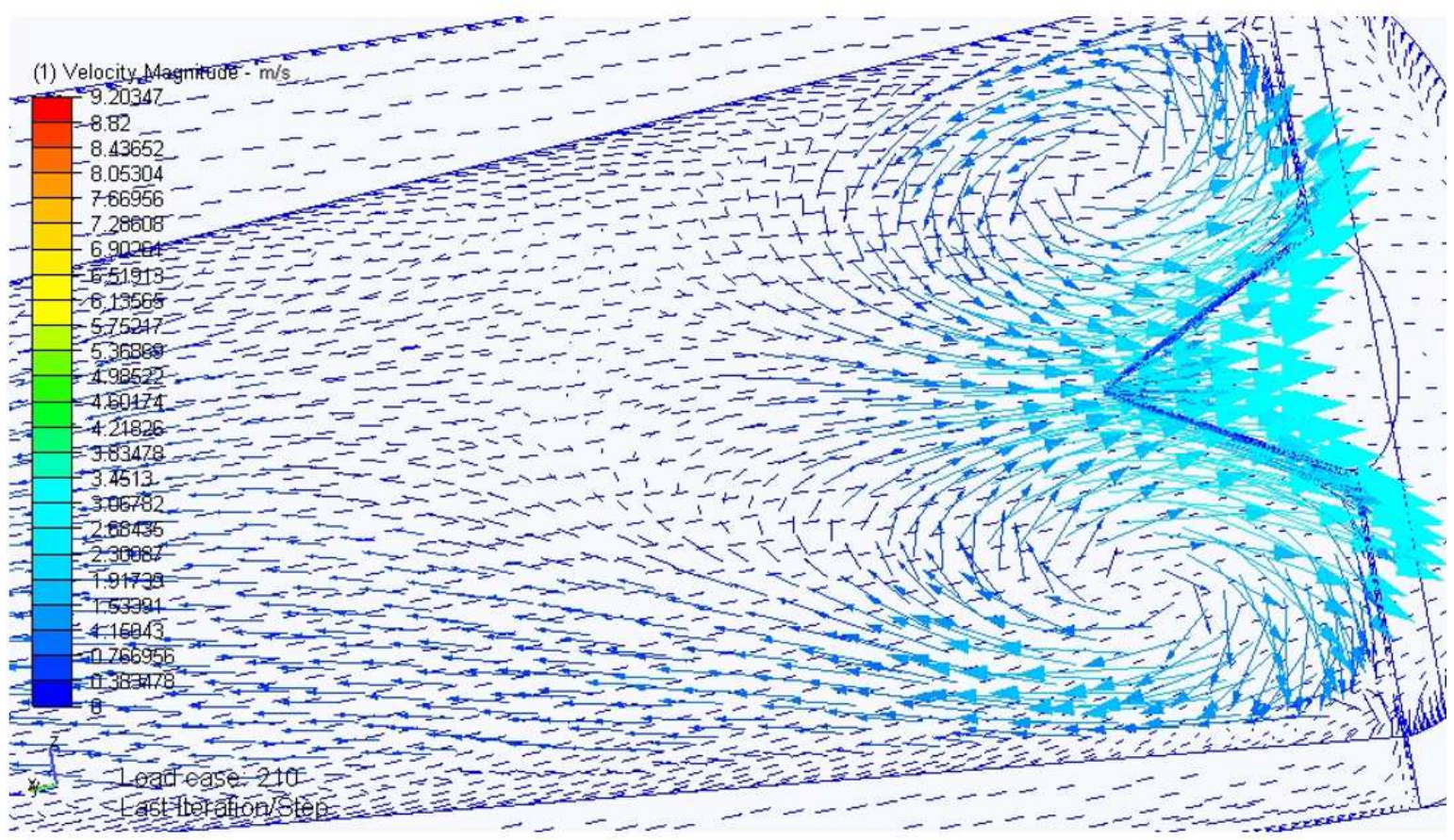

Figure 7. The occurrence of a vortex ring near the condensation surface inside of Laval-liked HPs at higher values heat loads entering to the HPs evaporator. In this case, the vortex ring formed from the center to the periphery of the vapour channel.

The numerical analysis of flow in the Laval-liked HPs condensation zone shows that the vortex structure has a spatial nature, at the same time the flow asymmetry becomes apparent being determined by non-linear friction against the underlying surface and the two-dimensional compressibility of the wet condensing vapour as well. Overheating of the evaporator in reference to the boiling temperature of diethyl ether at atmospheric pressure is $15 \mathrm{~K}$, the temperature difference between the evaporator and the condensation surface is equal to $25 \mathrm{~K}$.

\subsection{The Distribution of the Axial Component of Velocity}

In the Figure 8 shows the results of numerical analysis of the distribution of the longitudinal component of the velocity of compressible moist vapour within the Laval-like HP at high heat loads. The formation of a vortex ring wet condensing vapour near the condensation surface inside of Laval-liked HPs leads to quite interesting results. Vortex ring 
is a highly gradient zone of the condensable moist vapour velocity with the opposite directions inside and outside the vortex ring. The numerical analysis of the velocity distribution along the centerline of the vapour channel in the Laval-liked HPs including the vortex ring shows the occurrence of two positive peaks of the velocity, one of them is in the critical section of the nozzle and the other near the condensation surface. In a critical section of the nozzle the axial component of velocity reaches $85 \mathrm{~m} / \mathrm{s}$, and near the condensation surface the counter-flow reaches the velocity of $33 \mathrm{~m} / \mathrm{s}$. The distribution of the axial component of velocity of the moist vapour shows the presence of a counter current due to the formation of a ring vortex within the vapor channel near the HPs condensation surface. This figure of the velocity distribution, shown in Figure 8, confirms the fact that the vortex ring is a zone of sharp velocity gradients, and as a consequence is also a pressure gradient. In the central part the vortex ring has a noticeable positive dynamic pressure, and it has a negative dynamic pressure in the peripheral part of the vortex ring. This means that lower static pressure occurs in the central part of the vortex ring and this leads to additional absorption of moist vapor in the condensation zone of Laval-liked HPs.
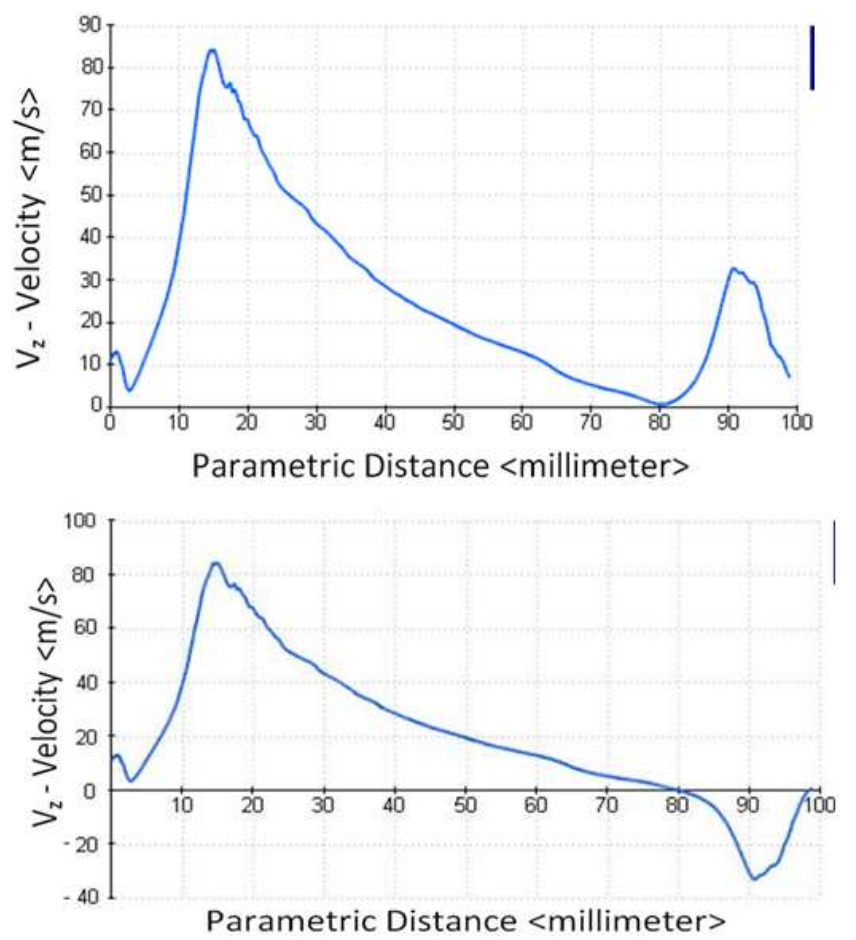

Figure 8. The distribution of the axial component of velocity of the moist vapour inside the vapour channel of Laval-liked HPs along the HPs centerline. Upper pattern with a positive velocity peak near the condensation surface of Laval-liked HPs represents the velocity distribution along the longitudinal axis of vapour channel and inside the vortex ring. The figure on the bottom with a negative velocity value peak near the surface of condensation represents the velocity distribution along the longitudinal axis and outside of a vortex ring and shows the presence of a counter current due to the formation of a ring vortex near the HPs condensation surface. Overheating of the evaporator in reference to the boiling temperature of diethyl ether at atmospheric pressure is $15 \mathrm{~K}$, the temperature difference between the evaporator and the condensation surface is equal to $25 \mathrm{~K}$.
Apparently, this means that the static pressure in the vortex center in the Laval - liked HP is less than the pressure in the axial part of the condensation zone of the HP with standard cylindrical vapor channel. The appearance of high static pressure in the peripheral part of the vortex ring, where there is a negative dynamic pressure leads to intensification of the condensation process near the top cap of the Laval-liked HPs, which leads, apparently, to an increased value of heat transfer coefficient in the Laval-liked HPs. This explains the reason why the heat transfer coefficient of Laval - like HPs becomes greater than HPs with the cylindrical vapor channel at boiling point in evaporator HP and there is formation of large amounts of vapor and increase in the efficiency of the nozzle in comparison with a cylindrical channel.

\section{Measurement of Pulse Characteristics of HPs}

The pulsation characteristics of HPs were measured in the following way [14-15]. An overheating was imposed on the evaporator by $\delta \mathrm{T}=\mathrm{T}-\mathrm{T}_{\mathrm{B}}$ relative to the boiling temperature of diethyl ether at atmospheric pressure with the aid of resistive heater 6 and high-precision temperature controller increased in discrete steps of $1 \mathrm{~K}$. Electrical pulses of 10-100 $\mathrm{kHz}$ and amplitude $5 \mathrm{~V}$ are applied to the electrodes of the capacitive sensor 10, Figure 4. The modulation rate is measured via a gain and filtering circuit 13, whereupon the signal goes to measuring input of digital oscilloscope 14 . The oscilloscope is connected to the computer 15 via USBinterface. Started from the specific overheating $\delta \mathrm{T}_{\mathrm{S}}=(\mathrm{T}-$ $\mathrm{T}_{\mathrm{B}}$ ) of HPs evaporator, electrical pulses become modulated. The modulation rate is measured using gain and filtering circuit 13, digital oscilloscope 14 and computer 15, and are shown in Figure 4. Measuring inaccuracy of modulation rate does not exceed 3-5 Hz.

Table 1 shows the pulsation rate values (modulation rate), obtained in the Laval-liked HP, $\mathrm{f}_{1}[\mathrm{~Hz}]$, and in the HP with standard cylindrical vapour channel, $\mathrm{f}_{2}[\mathrm{~Hz}]$, depending on overheating $\delta \mathrm{T},[\mathrm{K}]$, of the evaporators.

Table 1. Pulsation rate values.

\begin{tabular}{lll}
\hline $\begin{array}{l}\text { Overheating of the } \\
\text { evaporator, } \boldsymbol{\delta} \mathbf{T}, \mathbf{K}\end{array}$ & $\begin{array}{l}\text { Pulsation frequency } \\
\mathbf{f}_{\mathbf{1}}, \mathbf{H z}\end{array}$ & $\begin{array}{l}\text { Pulsation } \\
\text { frequency } \mathbf{f}_{\mathbf{2}}, \mathbf{H z}\end{array}$ \\
\hline 9.05 & $386 \pm 5$ & - \\
10.1 & $396 \pm 5$ & - \\
11.03 & $426 \pm 5$ & $406 \pm 5$ \\
12.15 & $450 \pm 5$ & $420 \pm 5$ \\
13.0 & $456 \pm 5$ & $437 \pm 5$ \\
14.07 & $474 \pm 5$ & $440 \pm 5$ \\
15.03 & $474 \pm 5$ & $454 \pm 5$ \\
16.0 & $478 \pm 5$ & $453 \pm 5$ \\
17.1 & $490 \pm 5$ & $460 \pm 5$ \\
18.06 & $491 \pm 5$ & $472 \pm 5$ \\
19.02 & $495 \pm 5$ & $473 \pm 5$ \\
20.12 & $502 \pm 5$ & $474 \pm 5$ \\
\hline
\end{tabular}

Both HPs have equal outer diameters $20 \mathrm{~mm}$ and equal sectional areas of capillary-porous inserts near the condensation region. As the overheating of the evaporator 
$\delta \mathrm{T} \sim 20 \mathrm{~K}$ increases, pulsation frequency in the Laval-liked nozzle goes up to $\sim 502 \mathrm{~Hz}$, derivative of the relationship between pulsation frequency and temperature is approximately $10.5 \mathrm{~Hz} / \mathrm{K}$. While investigating $\mathrm{HPs}$ with standard cylindrical vapour channel and equal outer diameter
$20 \mathrm{~mm}$, length $100 \mathrm{~mm}$ and thickness of the evaporator and capillary-porous insert $3 \mathrm{~mm}$, initial pulsed flows occur in event of overheating of the evaporator $\delta \mathrm{T} \sim 11 \mathrm{~K}$, frequency (modulation frequency of electromagnetic pulsation) $\mathrm{f} \sim 406$ $\mathrm{Hz}$.

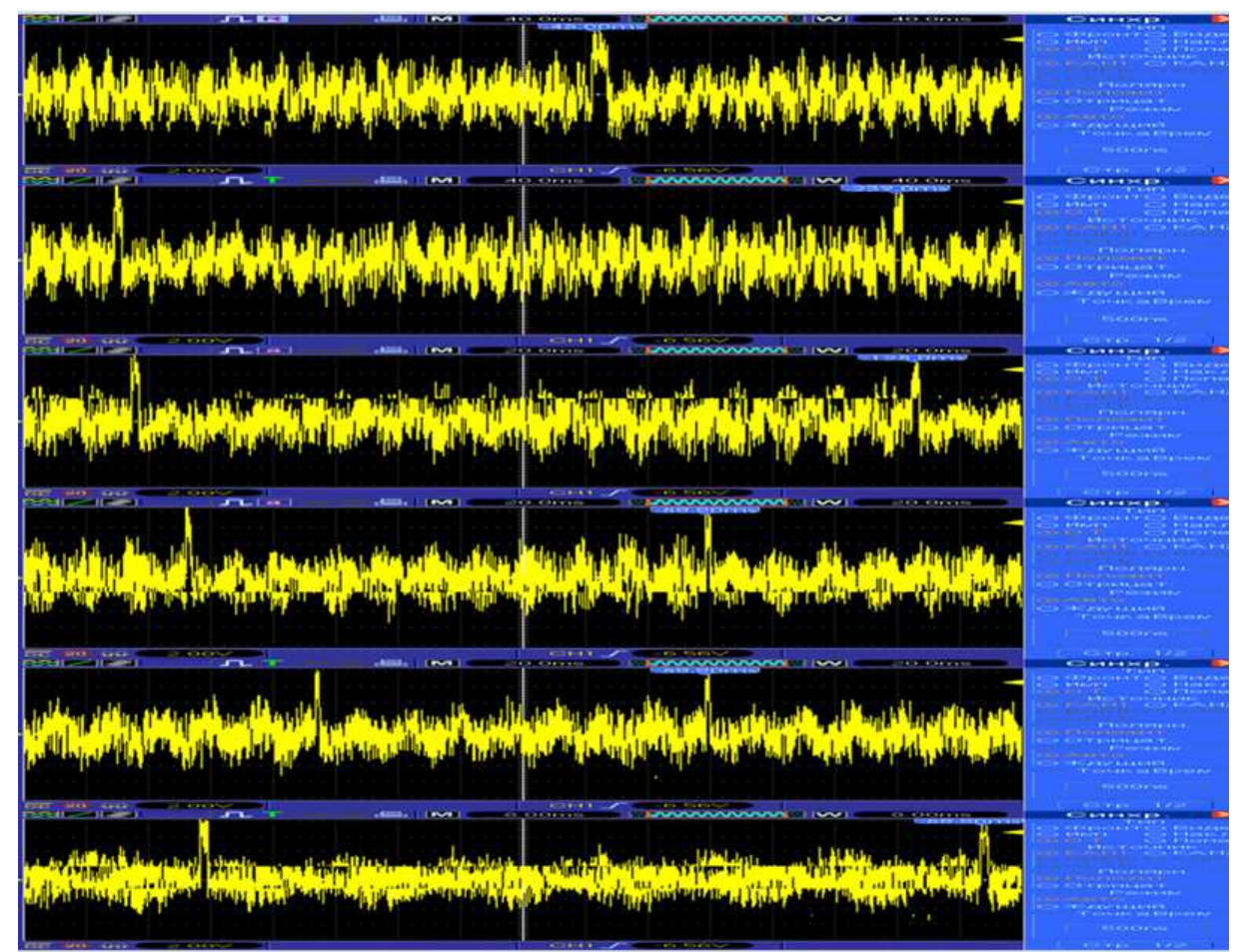

Figure 9. Oscillograms of the increase of the modulation frequency depending on the thermal load on Laval-liked HPs. Initial pulsed flows occur in the Lavalliked HPs in the event of overheating of the evaporator $\delta T \sim 9 K$, frequency (modulation frequency of electromagnetic pulsation) is $f_{1} \sim 386 H z$, (lower oscillogram); $\delta T \sim 20 \mathrm{~K} f_{1} \sim 502 \mathrm{~Hz}$, (upper oscillogram). The oscillograms without pulsations in the vapour channel of HPs does not have a low-frequency (400-500) Hz modulating features.

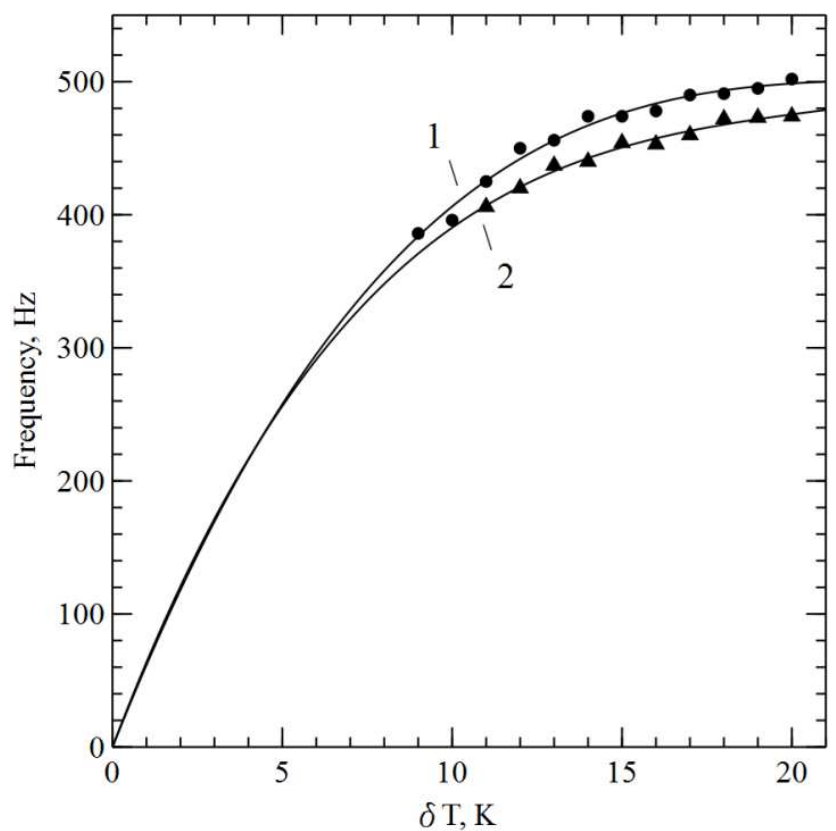

Figure 10. Test values of modulation frequency depending on overheating $\delta T$ $=\left(T-T_{B}\right)$ of HPs evaporator, with reference to the boiling temperature of diethyl ether $308.65 \mathrm{~K}\left(35.5^{\circ} \mathrm{C}\right) .1$ - HP with the Laval-liked vapour channel; 2- HP with a standard cylindrical vapour channel.
As the overheating of the evaporator $\delta \mathrm{T} \sim 20 \mathrm{~K}$ increases, pulsation frequency in the cylindrical vapour channel goes up to $474 \mathrm{~Hz}$, derivative of the relationship between pulsation frequency and temperature is approximately $7.5 \mathrm{~Hz} / \mathrm{K}$.

The insensitivity zone of the capacity sensors in the cylindrical channel, defined by the initial convective nature of the vapour flow, is greater than in the vapour channel of Laval-liked HPs. Dynamic range of pulsations in the Lavalliked HPs vapour channel, is a little greater in frequency, in comparison to dynamic range of pulsations $406 \mathrm{~Hz}-474 \mathrm{~Hz}$ in cylindrical vapour channel, and equals to $386 \mathrm{~Hz}-502$ $\mathrm{Hz}$. Measuring inaccuracy does not exceeds 3-5 Hz.

\subsection{Numerical Model Details}

Numerical simulations of the vortex pulsation flows inside a vapour channel of the Laval -liked HP have been performed in finite element modeling in CFD 10.0 code Fluent 6.3.26 under 2D, double precision axi-symmetric conditions. Navier-Stokes equations with measured boundary conditions were solved, i.e. using fixed temperature values of heat source and heat outlet. The model was studied as a longitudinal section along the axes of the two injector channels, which helps to preserve all the specific features of whirling instability under the conditions of continuous 
circulation motion of the working fluid during liquid and vapour phases. In the construction of the design model about 457233 finite elements were used, with increased meshing at injection capillary channels sections, nozzle throat section and turbulence element. The model size is a compromise between available computer resources and computational investigation error.

Is clearly visible transition from stationary convective flow regime in the vapour channel to pulsatile flow regime. At high heat capacity, received by the HPs, and boiling in the grid evaporator, excessive vapour occurs in the convergent part of the nozzle, thus leads to pressure increase up to the
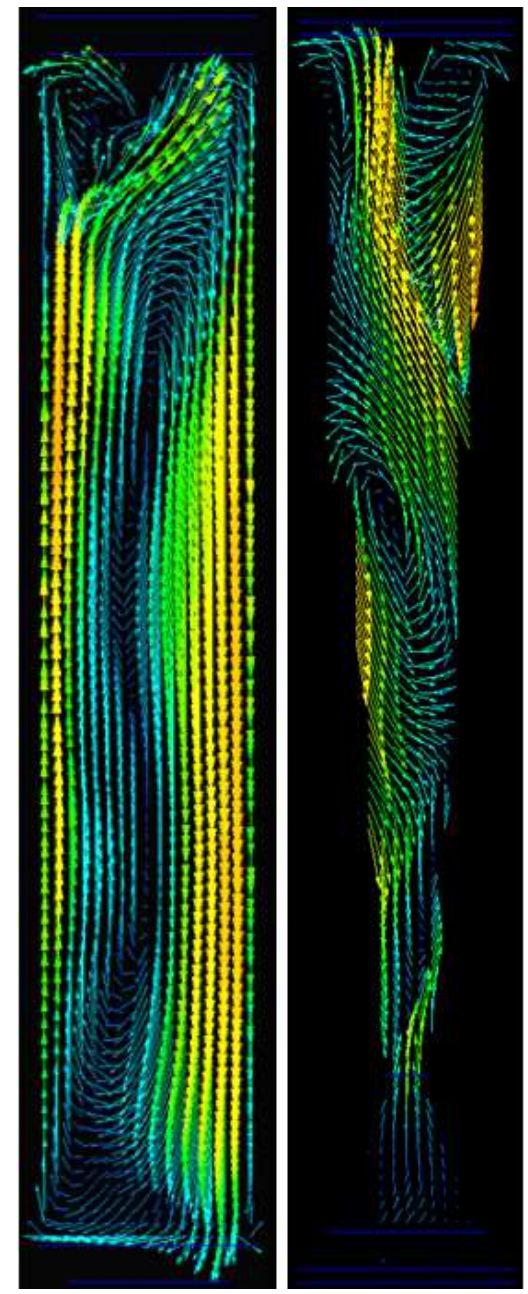

Figure 11. The results of flow simulation of compressible supersaturated vapour environment inside a vapour channel. The figure presents test values of the vapour flow pulsation in the vapour channel in the Laval-liked form of a HP, as overheating of HPs evaporator is increased in reference to boiling temperature of diethyl ether $308.55 \mathrm{~K}$ by $2 \mathrm{~K} ; 5 \mathrm{~K} ; 8 \mathrm{~K}$; and $13 \mathrm{~K}$, from left to the right.

\subsection{Validation of Numerical Scheme}

Evident conversion from convection mode to convectionvortex mode and then to pulsation mode of the vapour flow inside of the HP, is recorded at evaporator overheating value $\delta \mathrm{T} \sim 9 \mathrm{~K}$.

Maximum value of velocity of diethyl ether moist vapour flow, obtained using calculation method in the throat section of the vapour channel using by the colour indication of the software CFD Design 10.0, reaches $100-110 \mathrm{~m} / \mathrm{s}$ when
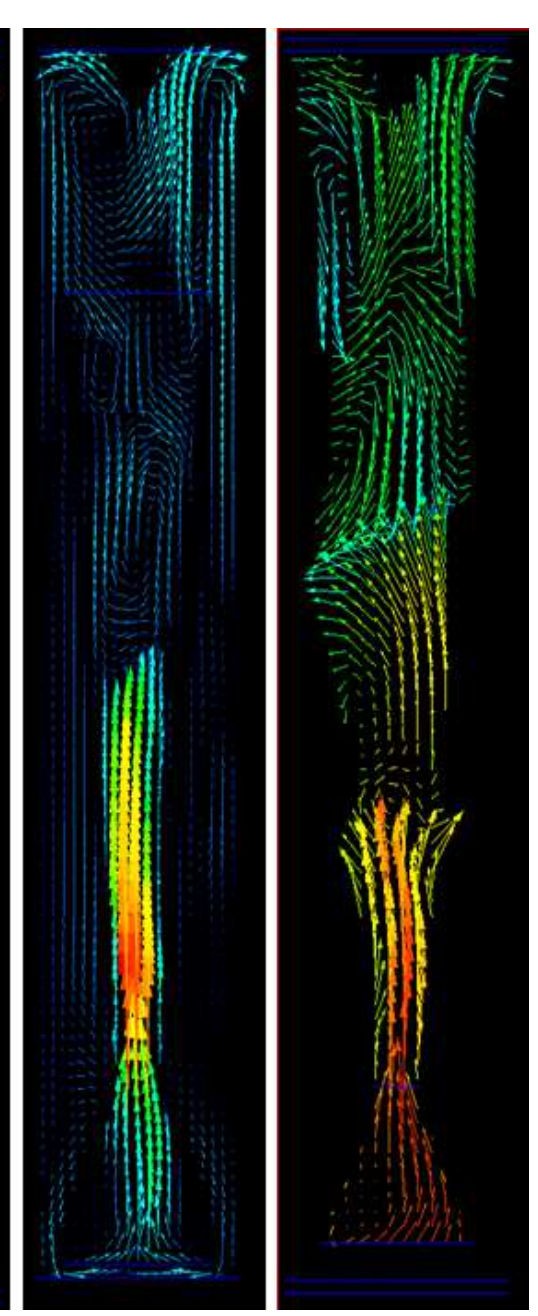

value, at which the average temperature of layers of the flat grid evaporator becomes lower than the boiling temperature working fluid, and boiling in the evaporator stops. Excessive vapour spreads through the divergent part of the vapour channel to the cooled area of the HP and is partially condensed. Due to the condensation effect, the pressure in the vapour channel decreases and the boiling process in the flat evaporator proceeds. Pressure increase period, vapour wave spreading in the condensation area of the HP and return expansion wave define the pulsation period in the vapour channel. 
section of the vapor channel $\mathrm{u}_{\mathrm{vp}} \sim(100-110) \mathrm{m} / \mathrm{s}$; critical diameter of the vapour channel $\mathrm{D}_{\mathrm{C}} \sim 4 \cdot 10^{-3} \mathrm{~m}$, and obtain the value $\operatorname{Re} \sim(1.5-1.65) \cdot 10^{5}$, the Prandtl number $\operatorname{Pr}=0.77$.

Duration of the pulsation period $\Delta \tau_{0}$ inside the divergent part of the $\mathrm{HP}_{\mathrm{S}}$ vapour channel can be estimated using the formula:

$$
\Delta \tau_{0} \sim \frac{\Delta l}{u_{v p}}
$$

Then substitute values of distance between pulsation crests (maximums) obtained in Figure $11 \Delta \mathrm{l} \sim(2-3) \cdot 10^{-2} \mathrm{~m}$, moist vapour flow velocity in divergent part of the vapour channel obtained using colour indication by the software CFD Design $10.0 \mathrm{u}_{\mathrm{vp}} \sim(20-30) \mathrm{m} / \mathrm{s}$, and you will obtain numerical value of duration of the pulsation $\Delta \tau_{0} \sim(0.75-1.5) \cdot 10^{-3} \mathrm{~s}$.

\section{Analytical Evaluation}

Evaporation cycle in HPs exist at low heat load of the evaporator, up to 4-6 W/ $/ \mathrm{cm}^{2}$, and is characterized by convective flow in the vapour channel. Evaporation cycle of short HPs, when evaporator heating power is constant and maximum value is limited to prevent the development of bubble boiling in the flat grid evaporator, is defined in the following way:

$$
\mathrm{E}=\frac{\Delta \mathrm{Q}}{\Delta \tau}<\mathrm{E}_{\mathrm{B}}
$$

where $\Delta \mathrm{Q}$ - heat energy, absorbed in the evaporator over a period of $\Delta \tau, \mathrm{J} ; \Delta \tau$ - time, sec; $\mathrm{E}_{\mathrm{B}}$ - thermal power, wherein the process of bubble boiling begins in the grid evaporator $\mathrm{W}$.

Rate of evaporation of dry monomolecular vapour over the evaporator is defined by the following equation:

$$
\dot{\mathrm{M}}=\dot{\mathrm{n}}_{\mathrm{vp}} \mathrm{m}_{\mathrm{vp}}=\frac{\mathrm{E}}{\mathrm{r}\left(\mathrm{T}_{\mathrm{B}}\right)}
$$

where $\dot{M}$ - the amount of the dry vapour, generated over the evaporator per unit time, $\mathrm{kg} / \mathrm{s} ; \dot{\mathrm{n}}_{\mathrm{vp}}$ - growth velocity of the number of vapour molecules over the evaporator per unit time, $\mathrm{s}^{-1} ; \mathrm{m}_{\mathrm{vp}}$ - mass of molecule of diethyl ether, $\mathrm{kg} ; \mathrm{r}\left(\mathrm{T}_{\mathrm{B}}\right)$ specific vaporization heat of the diethyl ether in HPs, depend upon temperature and pressure, $\mathrm{J} / \mathrm{kg} ; \mathrm{T}_{\mathrm{B}}(\mathrm{P})$ - boiling temperature of the diethyl ether, $\mathrm{K}$.

Mass flow of saturated dry monomolecular vapour over the evaporator is defined by the following equation:

$$
\mathrm{G}_{\mathrm{vp}}=\dot{\mathrm{M}}=\mathrm{F}(z) \rho_{\mathrm{vp}}\left(\mathrm{T}_{\mathrm{ev}}\right) \mathrm{u}_{\mathrm{vp}}=\frac{\mathrm{E}}{\mathrm{r}\left(\mathrm{T}_{\mathrm{B}}\right)}
$$

where $G_{V P}$ - mass flow of dry saturated vapour over the evaporator, $\mathrm{kg} / \mathrm{s} ; \mathrm{n}_{\mathrm{vp}}\left(\mathrm{T}_{\mathrm{ev}}\right)$ - average number of dry vapour molecules in the unit volume over the evaporator, $\mathrm{m}^{-3} ; \mathrm{u}_{\mathrm{vp}}$ average velocity of the vapour phase, $\mathrm{m} / \mathrm{s} ; \rho_{\mathrm{VP}}\left(\mathrm{T}_{\mathrm{ev}}\right)$ - vapour density of diethyl ether over the evaporator, $\mathrm{kg} / \mathrm{m}^{3} ; \mathrm{T}_{\mathrm{ev}}-$ temperature of the evaporator, $\mathrm{K}$.

Growth rate of the number of molecules of vapour over the evaporator in the convergent region of the nozzle of the vapour channel, which defines excess pressure over the evaporator and mass flow of vapour in HPs, is calculated from the equation:

$$
\dot{\mathrm{n}}_{\mathrm{vp}}=\frac{\mathrm{E}}{\mathrm{r}\left(\mathrm{T}_{\mathrm{B}}\right) \mathrm{m}_{\mathrm{vp}}}=\frac{\mathrm{EN}_{\mathrm{A}}}{\mathrm{r}\left(\mathrm{T}_{\mathrm{B}}\right) \mu_{\mathrm{vp}}}
$$

where $\mathrm{N}_{\mathrm{A}}$ - Avogadro constant, $\mathrm{mol}^{-1} ; \mu_{\mathrm{vp}}$ - molar mass of diethyl ether vapour, $\mathrm{kg} / \mathrm{mol}$.

Assuming approximately equal velocities and without considering the jet type nature of the flow pattern, the linear velocity of the hydrodynamic flotation of the vapour flow over the evaporator surface is calculated from the equation:

$$
\mathrm{u}_{\mathrm{vp}} \simeq \frac{\dot{\mathrm{n}}_{\mathrm{vp}}}{\mathrm{F}(\mathrm{z}) \mathrm{n}_{\mathrm{vp}}\left(\mathrm{T}_{\mathrm{ev}}\right)}=\frac{\mathrm{EN}_{\mathrm{A}}}{\mathrm{F}(\mathrm{z}) \mathrm{r}\left(\mathrm{T}_{\mathrm{B}}\right) \mu_{\mathrm{vp}} \mathrm{n}_{\mathrm{vp}}\left(\mathrm{T}_{\mathrm{ev}}\right)}
$$

Insert expressions (9) and (8) in the equation (7) to obtain the formula for calculation of molecular flow of the dry vapour over the evaporator:

$$
G_{\mathrm{vp}} \simeq \rho_{\mathrm{VP}}\left(\mathrm{T}_{\mathrm{ev}}\right) \frac{\mathrm{EN}_{\mathrm{A}}}{\mathrm{r}\left(\mathrm{T}_{\mathrm{B}}\right) \mu_{\mathrm{vp}} \mathrm{n}_{\mathrm{vp}}\left(\mathrm{T}_{\mathrm{ev}}\right)}
$$

Hydrodynamic flow of saturated dry vapour is defined by pressure difference value between the evaporator and condensation region in HP, according to the formula:

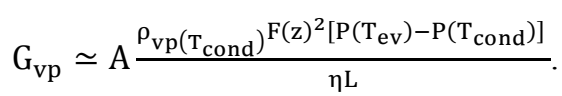

where A - nondimensional constant about a unit; $\eta$ coefficient of dynamic viscosity of dry vapour, $\mathrm{Pa} \cdot \mathrm{s} ; \mathrm{L}-$ the length of the vapour channel of the HPs, $\mathrm{m} ; \mathrm{P}\left(\mathrm{T}_{\mathrm{ev}}\right)$ - vapour pressure under the HPs evaporator, $\mathrm{Pa} ; \mathrm{P}\left(\mathrm{T}_{\text {cond }}\right)$ - vapour pressure near the condensation surface of the HPs, $\mathrm{Pa} ; \Delta \mathrm{P}_{\mathrm{vp}}$ vapour pressure difference over the evaporator and near the condensation surface in the vapour channel of the HPs, Pa; $\mathrm{T}_{\text {cond }}$ - temperature of the condensation surface, $\mathrm{K}$.

Equate mass and hydrodynamic flow of saturated dry vapour, and obtain the following equation:

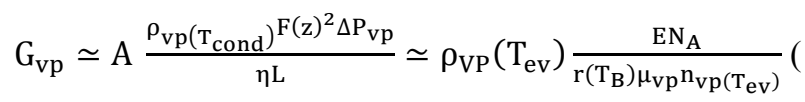

Excess pressure over the evaporator defines transfer of the vapour flow in the vapour channel of the HPs, and is calculated in linear approximation according to the equation:

$$
\mathrm{P}\left(\mathrm{T}_{\mathrm{ev}}\right) \simeq \mathrm{P}\left(\mathrm{T}_{\text {cond }}\right)+\frac{\mathrm{dP}}{\mathrm{dT}}\left(\mathrm{T}_{\mathrm{ev}}-\mathrm{T}_{\text {cond }}\right)
$$

With respect to the Clapeyron-Clausius equation, pressure derivative of the vapour by temperature is calculated in a conventional manner, nevertheless considering the fact that for liquid the specific volume ratio is small, $v^{\mathrm{L}} / v^{\mathrm{VP}}<10^{-2}-$ $10^{-3}$, hence in the Clapeyron-Clausius equation the value of the specific volume of fluid $v^{\mathrm{L}}$ is ignored, and in ideal gas state the following equation is obtained:

$$
\frac{\mathrm{dP}}{\mathrm{dT}}=\frac{1}{\mathrm{~T}} \frac{\mathrm{r}\left(\mathrm{T}_{\mathrm{B}}\right)}{\left(\mathcal{V}^{\mathrm{VP}}-\mathcal{V}^{L}\right)} \simeq \frac{\mathrm{r}\left(\mathrm{T}_{\mathrm{B}}\right)}{\mathrm{T}_{\text {cond }}} \rho_{\mathrm{vp}}\left(\mathrm{T}_{\text {cond }}\right)
$$

where $v^{\mathrm{VP}}$ - specific volume of the saturated vapour, $\mathrm{m}^{3} / \mathrm{kg}$; $v^{\mathrm{L}}$ - specific volume of the diethyl ether on the liquid- 
vapour coexistence line, $\mathrm{m}^{3} / \mathrm{kg}$.

Substitute expression (10) in (8), and obtain the equation to calculate vapour quantity in the HP:

$$
\frac{\mathrm{F}(\mathrm{z})^{2}}{\eta \mathrm{L}} \frac{\mathrm{r}\left(\mathrm{T}_{\mathrm{B}}\right)}{\mathrm{T}_{\text {cond }}} \rho_{\mathrm{vp}}\left(\mathrm{T}_{\mathrm{ev}}-\mathrm{T}_{\text {cond }}\right) \simeq \frac{\mathrm{EN}_{\mathrm{A}}}{\mathrm{r}\left(\mathrm{T}_{\mathrm{B}}\right) \mu_{\mathrm{vp}} \mathrm{n}_{\mathrm{vp}\left(\mathrm{T}_{\mathrm{ev}}\right)}}
$$

Vapour temperature over the evaporator's surface with low evaporation and without boiling is defined by the equation (16):

$$
\frac{\rho_{\mathrm{vp}}\left(\mathrm{T}_{\text {cond }}\right) \mathrm{F}(\mathrm{z})^{2} \mathrm{r}\left(\mathrm{T}_{\mathrm{B}}\right)^{2} \mu_{\mathrm{vp}} \mathrm{n}_{\mathrm{vp}\left(\mathrm{T}_{\text {cond }}\right)}}{\mathrm{N}_{\mathrm{A}} \eta \mathrm{LT} \mathrm{T}_{\text {cond }}}\left(\mathrm{T}_{\mathrm{ev}}-\mathrm{T}_{\text {cond }}\right) \simeq \mathrm{K}_{\mathrm{HP}} \Delta \mathrm{T}
$$

where $\mathrm{n}_{\mathrm{vp}}\left(\mathrm{T}_{\text {cond) }}\right.$ - average number of dry vapour molecules in the unit volume near the condenser, $\mathrm{m}^{-3}$;

Heat transfer coefficient $\mathrm{K}_{\mathrm{HP}}$ at cross-section of the vapour channel of the HP is defined according to the following expression:

$$
\mathrm{K}_{\mathrm{HP}} \simeq \frac{\rho_{\mathrm{vp}}\left(\mathrm{T}_{\text {cond }}\right) \mathrm{F}(\mathrm{z})^{2} \mathrm{r}\left(\mathrm{T}_{\mathrm{B}}\right)^{2} \mu_{\mathrm{vp}} \mathrm{n}_{\mathrm{vp}\left(\mathrm{T}_{\text {cond }}\right)}}{\mathrm{N}_{\mathrm{A}} \eta \mathrm{LT}_{\text {cond }}}
$$

When the rate of heat supply into the thin evaporator is high, its average temperature is greater than the boiling temperature of the working fluid during bubble boiling or vaporization. The hydrodynamic vapour flow at the converging region of the nozzle has no time to carry out heating capacity generated through boiling in the evaporator.

Vapour density and pressure increase and boiling temperature of the working fluid goes up until it exceeds average temperature of the evaporator. Upon pressure increase the boiling in the evaporator stops (slows), and an overpressure wave spreads in the vapour channel to the condensation region of the HPs, where the vapour becomes supersaturated, and condenses. The condensation process is not instant, and when the evaporator's activity is slowed (stopped), condensation lasts until the pressure decreases to the vapour saturation pressure at the condensation temperature, thereafter the condensation process stops.

The slow process of saturated vapour pressure decrease, due to condensation, feeds back through the vapour channel of the HP back to the evaporator, and the boiling process there is resumed.

\section{Discussion}

Pressure pulsations in the vapour channel of the short HPs result from boiling and intensive vapour generation in the evaporator, non-instantaneous mass-transfer through the vapour channel to the condensation region of the HP, and the slow condensation process, which provides pressure decrease in the condensation region at first and then in the evaporator of the HPs.

Following all described processes the next pulsed evaporation cycle is resumed. The heating capacity, entering to the flat grid evaporator of the short HP, when the evaporator's temperature exceeds the boiling temperature $T_{B}$ (p) of the working fluid, is defined according to the next

$$
\mathrm{T}_{\mathrm{ev}} \simeq \mathrm{T}_{\text {cond }}\left(1+\frac{\mathrm{EN}_{\mathrm{A}} \eta \mathrm{L}}{\rho_{\mathrm{vp}}\left(\mathrm{T}_{\text {cond }}\right) \mathrm{F}(\mathrm{z})^{2} \mathrm{r}\left(\mathrm{T}_{\mathrm{B}}\right)^{2} \mu_{\mathrm{vp}} \mathrm{n}_{\mathrm{vp}\left(\mathrm{T}_{\mathrm{ev}}\right)}}\right) \leq \mathrm{T}_{\mathrm{B}} .
$$

Steady-state evaporation conditions in HP mean that the temperature in the evaporator does not exceed boiling temperature of the working fluid. Heating capacity of the HP, $\mathrm{W}$, is defined according to the following equation: equation:

$$
E=\frac{\left[T_{e v}-T_{B}(p)\right] F(z)}{R_{e v}(T)}
$$

It is considered that moist vapour stream is formed of two subsystems: microdrops stream system $G_{d r}$ and dry vapour stream system, $\mathrm{G}_{\mathrm{vp}}$. Rate of vaporization of the moist droplet vapour flow is defined in the standard way:

$$
\dot{\mathrm{M}}=\mathrm{G}_{\mathrm{mix}}=\mathrm{G}_{\mathrm{vp}}+\mathrm{G}_{\mathrm{dr}}
$$

For the purpose of simplification of the analytical model construction, the real droplet vapour flow over the evaporator - having microdrops dimensioned by complicated doublehumped distribution function, Lee, Reges, Almenas, [30], is reported in terms of a mono-dispersal system of spherical microdrops with arithmetic middling radius $r_{a}$, which is frequently used while analyzing two-phase droplet vapour flows:

$$
\mathrm{r}_{\mathrm{a}}=\frac{1}{\mathrm{n}_{\mathrm{dr}}} \sum_{i=0}^{\infty} \mathrm{r}_{\mathrm{dri}} \mathrm{n}_{\mathrm{dri}}
$$

where $n_{d r}$ - total number of microdrops of all sizes per unit volume of the droplet vapour flow over the evaporator, $1 / \mathrm{m}^{3}$; $\mathrm{n}_{\mathrm{dri}}$ - number of microdrops of the diethyl ether with radius $r_{\text {dri }}$ per unit volume of the droplet vapour medium, $1 / \mathrm{m}^{3}$.

Considering the accepted assumption over spherical shape of microdrops, the expression for absolute moisture $\gamma$ of the droplet vapour flow is defined in the standard way:

$$
\gamma=\frac{\mathrm{M}_{\mathrm{dr}}}{\mathrm{M}_{\mathrm{dr}}+\mathrm{M}_{\mathrm{vp}}}=\left[1+\frac{\rho_{\mathrm{vp}}}{\rho_{L}}\left(\frac{3}{4 \pi} \frac{1}{\mathrm{r}_{\mathrm{a}}^{3} n_{\mathrm{dr}}}-1\right)\right]^{-1}
$$

where $\mathrm{M}_{\mathrm{dr}}$ - total mass of microdrops per unit volume of the droplet vapour flow over the evaporator, $\mathrm{kg} ; \mathrm{M}_{\mathrm{vp}}$ - vapour mass per unit volume of the dry vapour flow over the evaporator, $\mathrm{kg} ; \mathrm{n}_{\mathrm{dr}}$ - total number of microdrops of all sizes per unit volume of the droplet vapour flow over the evaporator, $1 / \mathrm{m}^{3} ; \mathrm{n}_{\mathrm{dr}}$ - total number of microdrops of all sizes per unit volume of the droplet vapour flow over the evaporator, $1 / \mathrm{m}^{3} ; \rho_{\mathrm{dr}}$ - density of microdrops of the diethyl ether, $\mathrm{kg} / \mathrm{m}^{3}$.

The density of moisture vapour $\rho_{\mathrm{vp}}^{\operatorname{mix}}\left(\mathrm{T}_{\mathrm{ev}}\right)$ without taking into account the relative motion phases is:

$$
\rho_{\mathrm{vp}}^{\operatorname{mix}}=\frac{\rho_{d r} \rho_{\mathrm{vp}}}{\gamma \rho_{\mathrm{vp}}+(1-\gamma) \rho_{\mathrm{dr}}}
$$


Synergies between consumable and absolute mass concentrations of microdrops $\gamma_{\mathrm{G}}$ or consumable and absolute moisture are defined according to the following relation:

$$
\gamma_{\mathrm{G}}=\frac{\gamma \psi}{(1-\gamma)+\gamma \psi} ; \psi=\frac{\mathrm{u}_{\mathrm{dr}}}{\mathrm{u}_{\mathrm{vp}}}
$$

The value $\gamma_{\mathrm{G}}$ represents the relation between quantity of the condensed droplet phase and total quantity of the twophase droplet vapour, and, considering the expression (22) is as follows:

$$
\gamma_{\mathrm{G}}=\left[1+\frac{\rho_{\mathrm{vp}}}{\rho_{L} \psi}\left(\frac{3}{4 \pi} \frac{1}{\mathrm{r}_{\mathrm{a}}^{3} n_{\mathrm{dr}}}-1\right)\right]^{-1}=\frac{\mathrm{G}_{\mathrm{dr}}}{\mathrm{G}_{\mathrm{mix}}}
$$

Mass flow rate of microdrops on the evaporator's surface are considered to be proportional to vaporization velocity and

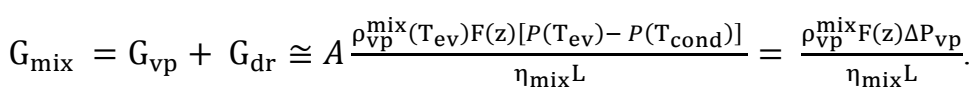

As a result of the boiling process in the evaporator, the pressure of the vapour over the evaporator increases up to $\mathrm{P}^{*}$, whereby the boiling process in the surface layers and further in the whole of the thin evaporator ( $3 \mathrm{~mm}$ thick) is slowed (stopped) due to the fact that average temperature of the evaporator $\mathrm{T}_{\mathrm{ev}}$ becomes lower than the boiling temperature $\mathrm{T}_{\mathrm{B}}$ of the working fluid in the evaporator under the increased pressure and the confined spaces:

$$
\mathrm{T}_{\mathrm{ev}}<\mathrm{T}_{\mathrm{B}}\left(\mathrm{P}^{*}\right)
$$

Furthermore, pulsing of the overpressured vapour begins to spread to the condensation region through the vapour channel. Cessation of boiling and retarding of vaporization in the evaporator of the HP lead to significant reduction (cessation) of the heat release and decrease in moist vapour transportation along the vapour channel of the HP to the condensation region of the HP. The time period $\Delta \tau_{\mathrm{ev}}$ of pressure increase up to $\mathrm{P}^{*}$ and cessation of boiling in the capillary-porous evaporator is estimated in linear approximation in ideal gas state and laminar heat transfer inside of the vapour channel of the HP, according to the formula:

$$
\Delta \tau_{\mathrm{ev}} \simeq \frac{\left[\mathrm{P}^{*}-\mathrm{P}\left(\mathrm{T}_{\text {cond }}\right)\right] \mathrm{F}(\mathrm{z}) \mathrm{L}}{\mathrm{k}_{\mathrm{B}} \mathrm{T}_{\mathrm{ev}} \dot{\mathrm{n}}_{\mathrm{vp}}} \simeq \frac{\left[\mathrm{P}^{*}-\mathrm{P}\left(\mathrm{T}_{\text {cond }}\right)\right] \mathrm{F}(\mathrm{z}) \operatorname{Lr}\left(\mathrm{T}_{\mathrm{B}}\right) \mathrm{m}_{\mathrm{vp}}}{\mathrm{Ek}_{\mathrm{B}} \mathrm{T}_{\mathrm{ev}}} .
$$

Surplus energy (increased pressure) release time $\Delta \tau_{\mathrm{HP}}$ in the evaporating region, by means of vapour flow transfer to the condensation region of the HP, is estimated according to the following formula:

$$
\Delta \tau_{H P} \simeq \frac{\mathrm{E} \Delta \tau_{\mathrm{ev}}}{\mathrm{r}\left(\mathrm{T}_{\mathrm{B}}\right) \rho_{\mathrm{vP}}^{\operatorname{mix}}\left(\mathrm{T}_{\mathrm{ev}}\right) \mathrm{u}\left(\mathrm{T}_{\mathrm{ev}}\right) \mathrm{F}(\mathrm{z})}
$$

Surplus pressure release time $\Delta \tau_{\mathrm{HP}}$ partially determines the time value of oscillations of the vapour flow in the vapour channel of the HP, during which the vapour pressure pulse, initiated over the evaporator, reaches the HP condensation surface, and condenses partially. The time period $\Delta \tau_{\text {cond }}$ of pressure decrease up to $\mathrm{P}\left(\mathrm{T}_{\text {cond }}\right)$ is estimated according to the mass flow rate of the vapour:

$$
\mathrm{G}_{\mathrm{dr}} \cong \mathrm{B}\left(\frac{\mathrm{E}}{\mathrm{r}\left(\mathrm{T}_{\mathrm{B}}\right)}\right)^{\mathrm{a}}\left(\frac{\mathrm{G}_{\mathrm{vp}}}{\mathrm{F}}\right)^{\mathrm{b}}
$$

where $\mathrm{a}$ and $\mathrm{b}$ - numerical coefficients; B - coefficient with account for thermophysical properties of the diethyl ether and structural parameters of the evaporator (porosity, typical dimension of channels and pores).

To evaluate the vapour quantity transported from the evaporator to the condensation region of the HP, equation (31) is used, where the microdrops subsystem contribution to the generation of overpressure of the two-phase droplet mixture over the evaporator $\mathrm{P}\left(\mathrm{T}_{\mathrm{ev}}\right)$ in the vapour channel is neglected:

formula:

$$
\Delta \tau_{\text {cond }} \simeq \frac{\mathrm{E} \Delta \tau_{\mathrm{ev}}}{\mathrm{r}\left(\mathrm{T}_{\text {cond }}\right) \rho_{\mathrm{vp}}^{\operatorname{mix}}\left(\mathrm{T}_{\text {cond }}\right) \mathrm{u}\left(\mathrm{T}_{\text {cond }}\right) \mathrm{F}(\mathrm{z})}
$$

As a result of the liquid phase formation, pressure near the cooling condensation surface reduces to:

$$
\mathrm{P}^{*} \simeq \mathrm{P}\left(\mathrm{T}_{\text {cond }}\right)
$$

This leads to the slowing of heat transfer through the vapour channel, the rarefaction wave propagation from the condensation zone to the evaporator and the start of the next cycle of pulsed increase of the pressure near the evaporator's surface. Thus, cycle pulse duration $\Delta \tau_{0}$ in the vapour channel of the HP:

$$
\Delta \tau_{0} \simeq \Delta \tau_{\mathrm{ev}}+\Delta \tau_{\mathrm{HP}}+\Delta \tau_{\text {cond }}+\Delta \tau_{\text {sound }}
$$

The analysis of the obtained measured data of heat transfer over the evaporator shows that the designed HPs work in the boiling mode. This fact is confirmed by influences of operating parameters of the vaporization process (heat flow density $q$ and pressure $\mathrm{P}\left(\mathrm{T}_{\mathrm{ev}}\right)$ ) on the heat transfer coefficient $\alpha$. The influence of these parameters is related to the similar influence of heat flow density and pressure over the bubble boiling in the substantial volume. Performed numerical estimation of parts of pulsation duration $\Delta \tau_{0}$ from the expression (33) show the following.

Pressure increase duration $\Delta \tau_{\mathrm{ev}}$ over the HPs evaporator leads to increased values

$\Delta \tau_{\mathrm{ev}} \sim 10^{-1}-10^{-2} \mathrm{~s}$, which means incomplete adequate flat model for estimation of evaporability in the evaporator. It is necessary to consider nonequilibrium volumetric vaporization with regard to the structure and porosity of the evaporator, temperature and moisture gradient in the boiling two-phase working fluid.

Moving duration $\Delta \tau_{\mathrm{HP}}$ of the vapour cluster along the convergent-divergent HPs vapour channel at vapour flow velocity in the channel equal $\sim 100 \mathrm{~m} / \mathrm{s} \div 300 \mathrm{~m} / \mathrm{s}$, reaches $\Delta \tau_{\mathrm{HP}} \sim(4.8 \div 1.6) \cdot 10^{-3} \mathrm{~s}$, which is acceptable only in the case 
when sonic velocity in dry vapour is $\sim 300 \mathrm{~m} / \mathrm{s}$ and correspondingly large temperature gradients in the vapour channel.

Maximum duration of condensation period of moist vapour is also excessive and reaches $\Delta \tau_{\text {cond }} \sim 10^{-1}-10^{-2} \mathrm{~s}$

Duration of expansion wave motion from condensation region to the HP evaporator at sonic speed in moist vapour about $100 \mathrm{~m} / \mathrm{s}$, doesn't exceed $\Delta \tau_{\text {sound }} \sim 1 \cdot 10^{-3} \mathrm{~s}$.

Comparison of experimental measured results of pulsation period duration in the convergent-divergent vapour channel of short low temperature HPs $\Delta \tau_{\exp }=(2 \div 2.5) \cdot 10^{-3} \mathrm{~s}$ with the valued $\Delta \tau_{0} \sim(0.75-1.5) \cdot 10^{-3} \mathrm{~s}$ obtained using numerical method and the software CFD Design 10.0, shows that they have good fit values.

Comparison of experimental measured results of pulsation period duration in the convergent-divergent vapour channel of short low temperature HPs $\Delta \tau_{0}=(2 \div 2.5) \cdot 10^{-3}$ with estimated values obtained using analytical method, shows excessive values of analytical results. Suggested simple theoretical model of the flat surface evaporation gives excessive values of pulsation period duration and is not fully correspond to acutely nonequilibrium evaporation processes in the grid capillary porous evaporator with boiling working fluid.

\section{Conclusions}

1. Heat transfer coefficient of the short linear HPs with the Laval-liked vapour channel, reaches the value of $(22 \pm 1) \cdot 10^{3}$ $\mathrm{W} / \mathrm{m}^{2} \mathrm{~K}$; heat transfer coefficient of the HPs with the cylindrical vapour channel does not exceed $(15 \pm 1) \cdot 10^{3}$ $\mathrm{W} / \mathrm{m}^{2} \mathrm{~K}$, if thickness of the capillary-porous insert layer is 3 $\mathrm{mm}$, diameter $20 \mathrm{~mm}$ and length $100 \mathrm{~mm}$. Comparison of heat transfer coefficients in short HPs shows sufficient advantages of HP with the Laval-liked vapour channel in contrast with standard cylindrical channel.

2. The pulsatile flow regime in the vapour channel of short linear HPs occurs when the working fluid in the evaporator starts to boil. Vapour density and pressure increase and boiling temperature of the working fluid goes up until it exceeds average temperature of the evaporator. Upon pressure increase the boiling in the evaporator stops (slows), and an overpressure wave spreads in the convergentdivergent vapour channel to the condensation zone of the HPs, where the vapour becomes supersaturated, and condenses. Condensation of moist vapour results in pressure decrease in condensation region, and after arrival of the expansion wave at the HPs evaporator, pressure over the evaporator also decreases, the boiling process in the evaporator proceeds and the pulsation cycle in the vapour channel is repeated.

3. Flow stagnation leads to vortex formation, and their interaction causes pulse-coupled vortex decay, static pressure boost and complex reverse flows. Calculation results show that flow stagnation during the pulsation cycle leads to an enlargement of the recirculating region and augmentation of condensation, and this effect is significant.

With all the operating parameters of HPs, two effects were observed: pulsating flow regime of two-phase vapour flow and film-type condensation.

\section{References}

[1] Akachi H. Structure of Heat Pipe. US patent 1990. № 4921041.

[2] Tong B. Y., Wong T. N., Ooi K. T. Closed-loop pulsating heat pipe//Applied Thermal Engineering. 2001, v 21, № 18, pp. 1845-1862.

[3] Naik R., Varadarajan V., Pundarika G. and Narasimha K. R. Experimental Investigation and Performance Evaluation of a Closed Loop Pulsating Heat Pipe// Journal of Applied Fluid Mechanics, 2013. Vol. 6, №. 2, pp. 267-275.

[4] Bertossi R., Romestant C., Ayel V., Bertin Y. A theoretical study and review on the operational limitations due to vapour flow in heat pipes. Front heat pipes 3 (2012) 023001.

[5] Bandyopadhyay A., Majumdar A. Modeling of compressible flow with friction and heat transfer using the generalized fluid system simulation program (GFSSP) //Thermal Fluid Analysis Workshop (TFAWS) by NASA Glenn Research Center and Corporate College, Cleveland, September 10-14, 2007. pp. 114.

[6] Zhang Y. and A. Faghri, 2008, Advances and unsolved issues in pulsating heat pipes // Heat Transfer Engineering 2008, v. 29 (1), pp. 20-44.

[7] Qu W., Ma H. B. Theoretical Analysis of Startup of a Pulsating Heat Pipe, International Journal of Heat and Mass Transfer, vol. 50, pp 2309-2316, 2007.

[8] Kutz M. Pulsating Heat Pipes. Mechanical Engineers; Handbook, Energy and Power, Third Edition, Book 4, Chapter 9, 2006.

[9] H R Deng et al Experimental investigation on a pulsating heat pipe with hydrogen 2015 IOP Conf. Ser.: Mater. Sci. Eng. 101 012065 .

[10] Creatini F. et al. Pulsating Heat pipe Only for Space (PHOS): results of the REXUS 18 sounding rocket Campaign $2015 \mathrm{~J}$. Phys.: Conf. Ser. 655012042.

[11] Taft B. S., Williams A. D., Drolen B. L. Review of Pulsating Heat Pipe Working Fluid Selection // Journal of Thermophysics and Heat Transfer, 2012. v. 26, No. 4, pp. 651656.

[12] Seryakov A. V. Velocity measurements in the vapour channel of low temperature range heat pipes// International Journal of Engineering Research \& Technology 2013, v. 2, № 8, pp. 1595-1603.

[13] Gupta AK, Lilley, D., N. Sayred. Swirling flow. New York: Wiley. 1987. 588 p.

[14] Seryakov A. V. Pulsation flow in the vapour channel of low temperature range heat pipes// Direct Research Journal of Engineering and Information Technology 2014, v. 2 (1), pp. 110 .

[15] Seryakov A. V. Pulsation flow in the vapour channel of short low temperature range heat pipes // International Journal on Heat and Mass Transfer Theory and Application 2014, v. 2, № 2, pp. $40-49$. 
[16] Seryakov A. V., Ananiev V. I., Orlov A. V. Condensation research in the short low-temperature range heat pipes.//Proceedings of the $9^{\text {th }}$ Minsk International Seminar of Heat Pipes, Heat Pumps, Refrigerators, Power Sources. Minsk, Belarus, 7-10 September 2015, v 2. p. 168-176.

[17] Seryakov A. V., Ananiev V. I. Condensation research in the short low-temperature range heat pipes./Proceedings of the VIII International Symposium on Turbulence, Heat and Mass Transfer. Sarajevo, Bosnia and Herzegovina, September 15-18 2015. Begell House Inc. p. 693-696.

[18] Patent № 2431101 RF, F 28D 15/00/ Method of filling heat pipes. Seryakov A. V. Published 10. 10. 2011. Bulletin No. 28.

[19] Utility model patent No. 152108 / Capacitance sensor for determination of a fluid layer thickness / Seryakov A. V. Published on 27.06.2015. Bulletin No. 18/2015.

[20] Seryakov A. V., Konkin A. V., Belousov V. K. Application of a jet steam nozzle in medium-temperature range heat pipes // Bulletin of Siberian State Aerospace University. 2012. Issue 1 (41), pp. 142-147.

[21] Steinhart J. S., Hart S. R. Calibration curves for thermistors // Deep Sea Research and Oceanographic Abstracts. 1968, v. 15, № 4, p. 497-503.

[22] CORNERSTONE SENSORS. A, B, C Coefficients for Steinhart-Hart Equation. Temperature Sensors for Health, Science and Industry. 2010. 2p. California 92083. USA.
[23] Seryakov A. V. Temperature measurement with thermistors // Bulletin of Siberian State Aerospace University 2013, Issue 1 (47), pp. 167-172.

[24] Seryakov A. V. Improving the accuracy of temperature measurement with thermistors // Sensors and Systems 2013, №1, pp. 38-42.

[25] Seryakov A. V. A new method for temperature measurement using thermistors// International Journal of Engineering Research \& Technology 2013, v. 2, № 7, pp. 444-454.

[26] Seryakov A. V. A universal method for temperature measurement using thermistors// National Journal of Engineering and Technology Research. Academia Publishing. 2013, v. 1 (1), pp. 014-020.

[27] Faghri A. Heat Pipe Science and Technology. 1995. Washington USA, Taylor and Francis.

[28] Vargaftic N. B. Spravochnick po teplophizicheskim svoistvam gasov i zhidkostey. Moscow: Publishing house of Physico Mathematical literature. 1963. 708p.

[29] Tables of physical values. Guide under the editorship of Kikoin I. K., the member of Academy of Science. Moscow: Atomizdat 1976. 1008 p.

[30] Lee R., Reges J., Almenas K. Size and number density change of droplet populations above front during reflood // International Journal of Heat and Mass Transfer. 1984. v. 27. N4. p. 573-585. 